\title{
Approximation properties of bivariate complex $q$-Balàzs-Szabados operators of tensor product kind
}

\section{Esma Yıldız Özkan*}

\author{
"Correspondence: \\ esmayildiz@gazi.edu.tr; \\ esmayildiz@gmail.com \\ Department of Mathematics, \\ Faculty of Science, Gazi University, \\ Ankara, 06500, Turkey
}

\begin{abstract}
In this study, we consider the bivariate complex q-Balàzs-Szabados operators of the tensor product kind. Approximation properties of these operators attached to analytic functions on compact polydisks are investigated by using the results in the univariate case obtained for q-Balàzs-Szabados operators in (Ispir and Yıldız Özkan in J. Inequal. Appl. 2013:361, 2013). In this sense, the upper estimate, the Voronovskaja-type theorem, and the lower estimate are obtained. The exact degree of its approximation is also given.
\end{abstract}

MSC: $30 \mathrm{E} 10 ; 41 \mathrm{~A} 25$

Keywords: complex approximation; q-Balàzs-Szabados operators; order of convergence; Voronovskaja-type theorem; exact degree of approximation

\section{Introduction}

The approximation properties of the $q$-analogue operators in compact disks have recently been an active area of the research in the field of the approximation theory [1-8]. Details of the $q$-calculus can be found in [9-11].

Balázs [12] defined the Bernstein-type rational functions. She gave an estimate for the order of its convergence and proved an asymptotic approximation theorem and a convergence theorem concerning the derivative of these operators. In [13], Balázs and Szabados obtained the best possible estimate under more restrictive conditions, in which both the weight and the order of convergence would be better than [12]. They applied their results to the approximation of certain improper integrals by quadrature sums of positive coefficients based on a finite number of equidistant nodes. The $q$-form of these operators was given by Doğru. He investigated Korovkin-type statistical approximation properties of these operators for the functions of one and two variables [14]. Atakut and Ispir [15] defined the bivariate real Bernstein-type rational functions of the Bernstein-type rational functions given by Balázs in [12] and proved the approximation theorems for these functions. Ispir and Gupta [16] studied the Bézier variant of generalized Kantrovich-type Balazs operators.

Approximation properties of the rational Balázs-Szabados operators on compact disks in the complex plane were investigated by Gal [17]. He proved the upper estimate in an approximation of these operators. Also, he obtained the exact degree of its approximation 
by using a Voronovskaja-type result. In [18], the approximation properties given by Gal in the complex plane was extended to the bivariate case.

The complex $q$-Balázs-Szabados operators was defined in [19] as follows:

$$
R_{n}^{q}(f ; z)=\frac{1}{\prod_{s=0}^{n-1}\left(1+q^{s} a_{n} z\right)} \sum_{j=0}^{n} q^{j(j-1) / 2} f\left(\frac{[j]_{q}}{b_{n}}\right)\left[\begin{array}{l}
n \\
j
\end{array}\right]_{q}\left(a_{n} z\right)^{j},
$$

where $f: D_{R} \cup[R, \infty) \rightarrow \mathbb{C}$ is uniformly continuous and bounded on $[0, \infty)$ with $D_{R}=\{z \in$ $\mathbb{C}:|z|<R\}$ for $R>0, a_{n}=[n]_{q}^{\beta-1}, b_{n}=[n]_{q}^{\beta}, q \in(0,1], 0<\beta \leq \frac{2}{3}, n \in \mathbb{N}, z \in \mathbb{C}$, and $z \neq-\frac{1}{q^{s} a_{n}}$ for $s=0,1,2, \ldots$.

We consider the following complex bivariate $q$-Balázs-Szabados operators of the tensor product kind:

$$
R_{n, m}^{q_{1}, q_{2}}(f)\left(z_{1}, z_{2}\right)=\sum_{k=0}^{n} \sum_{j=0}^{m} f\left(\frac{[k]_{q_{1}}}{b_{n}}, \frac{[j]_{q_{2}}}{b_{m}}\right) p_{n, k}\left(z_{1}\right) p_{m, j}\left(z_{2}\right)
$$

where $f:\left(D_{R_{1}} \cup\left[R_{1}, \infty\right)\right) \times\left(D_{R_{2}} \cup\left[R_{2}, \infty\right)\right) \rightarrow \mathbb{C}$ is a uniformly continuous function bounded on $[0, \infty) \times[0, \infty), a_{n}=[n]_{q_{1}}^{\beta-1}, b_{n}=[n]_{q_{1}}^{\beta}, a_{m}=[m]_{q_{2}}^{\beta-1}, b_{m}=[m]_{q_{2}}^{\beta}$ for $n, m \in \mathbb{N}$, $q_{1}, q_{2} \in(0,1], 0<\beta \leq \frac{2}{3}$.

$$
p_{n, k}\left(z_{1}\right)=\frac{q_{1}^{k(k-1) / 2}\left[\begin{array}{l}
n \\
k
\end{array}\right]_{q_{1}}\left(a_{n} z_{1}\right)^{k}}{\prod_{s_{1}=0}^{n-1}\left(1+q_{1}^{s_{1}} a_{n} z_{1}\right)} \quad \text { and } \quad p_{m, j}\left(z_{2}\right)=\frac{q_{2}^{j(j-1) / 2}\left[\begin{array}{c}
m \\
j
\end{array}\right]_{q_{2}}\left(a_{m} z_{2}\right)^{j}}{\prod_{s_{2}=0}^{m-1}\left(1+q_{2}^{s_{2}} a_{m} z_{2}\right)}
$$

for all $s_{1}=0,1, \ldots, n-1, s_{2}=0,1, \ldots, m-1$ and $z_{1}, z_{2} \in \mathbb{C}$ with $z_{1} \neq-\frac{1}{q_{1}^{s_{1}} a_{n}}$ and $z_{2} \neq-\frac{1}{q_{2}^{s_{2}} a_{m}}$.

The complex bivariate $q$-Balázs-Szabados operators of the tensor product kind are well defined and linear, and these operators are analytic for all $n \geq n_{0}, m \geq m_{0},\left|z_{1}\right| \leq r_{1}<$ $\left[n_{0}\right]_{q_{1}}^{1-\beta}$ and $\left|z_{2}\right| \leq r_{2}<\left[m_{0}\right]_{q_{2}}^{1-\beta}$.

The aim of this paper is to obtain the exact degree of approximation of the complex bivariate $q$-Balázs-Szabados operators of the tensor product kind. The Voronovskaja-type theorem in the bivariate case is very different from the univariate case, so the exact degree of approximation of these operators can be obtained for $0<\beta<\frac{1}{2}$.

Throughout this paper, we denote by $\|f\|_{r_{1}, r_{2}}=\max \left\{\left|f\left(z_{1}, z_{2}\right)\right|:\left(z_{1}, z_{2}\right) \in \bar{D}_{r_{1}} \times \bar{D}_{r_{1}}\right\}$ the uniform norm of the function $f$ in the space of continuous functions on $\bar{D}_{r_{1}} \times \bar{D}_{r_{1}}$ and by $\|f\|_{B([0, \infty) \times[0, \infty))}=\sup \left\{\left|f\left(z_{1}, z_{2}\right)\right|:\left(z_{1}, z_{2}\right) \in[0, \infty) \times[0, \infty)\right\}$ the norm of the function $f$ in the space of bounded functions on $[0, \infty) \times[0, \infty)$, where $D_{r}=\{z \in \mathbb{C}:|z|<r\}$ for $r>0$.

The convergence results will be obtained under the condition that $f:\left(D_{R_{1}} \cup\left[R_{1}, \infty\right)\right) \times$ $\left(D_{R_{2}} \cup\left[R_{2}, \infty\right)\right) \rightarrow \mathbb{C}$ is analytic in $D_{R_{1}} \times D_{R_{2}}$ for $r_{1}<R_{1}$ and $r_{2}<R_{2}$, which ensures the representation $f\left(z_{1}, z_{2}\right)=\sum_{k=0}^{\infty} f_{k}\left(z_{2}\right) z_{1}^{k}$, where $f_{k}\left(z_{2}\right)=\sum_{j=0}^{\infty} c_{k, j} z_{2}^{j}$ for all $\left(z_{1}, z_{2}\right) \in D_{R_{1}} \times D_{R_{2}}$.

\section{Auxiliary results}

Let $q=\left(q_{n}\right)$ be a sequence satisfying

$$
\lim _{n \rightarrow \infty} q_{n}=1 \quad \text { and } \quad \lim _{n \rightarrow \infty} q_{n}^{n}=c \quad(0 \leq c<1) .
$$

We need the following lemmas in order to prove the main results for the operators (1). 
Lemma 1 Let $n_{0}, m_{0} \geq 2,0<\beta \leq \frac{2}{3}, \frac{1}{2}<r_{1}<R_{1} \leq \frac{\left[n_{0}\right]_{q_{1}}^{1-\beta}}{2}$ and $\frac{1}{2}<r_{2}<R_{2} \leq \frac{\left[m_{0}\right]_{q_{1}}^{1-\beta}}{2}$. If $f:$ $\left(D_{R_{1}} \cup\left[R_{1}, \infty\right)\right) \times\left(D_{R_{2}} \cup\left[R_{2}, \infty\right)\right) \rightarrow \mathbb{C}$ is a uniformly continuous function bounded on $[0, \infty) \times[0, \infty)$ and analytic in $D_{R_{1}} \times D_{R_{2}}$ then we have the form

$$
R_{n, m}^{q_{1}, q_{2}}(f)\left(z_{1}, z_{2}\right)=\sum_{k=0}^{\infty} \sum_{j=0}^{\infty} c_{k, j} R_{n, m}^{q_{1}, q_{2}}\left(e_{k, j}\right)\left(z_{1}, z_{2}\right)
$$

for all $\left(z_{1}, z_{2}\right) \in D_{r_{1}} \times D_{r_{2}}$, where $\left(e_{k, j}\right)\left(z_{1}, z_{2}\right)=e_{1}^{k}\left(z_{1}\right) e_{2}^{j}\left(z_{2}\right)$ with $e_{1}^{k}\left(z_{1}\right)=z_{1}^{k}, e_{2}^{j}\left(z_{2}\right)=z_{2}^{j}$ for $k, j \in \mathbb{N}$.

Proof For any $s, r \in \mathbb{N}$, we define

$$
\begin{aligned}
& f_{s, r}\left(z_{1}, z_{2}\right)=\sum_{k=0}^{s} \sum_{j=0}^{r} c_{k, j} e_{k, j}\left(z_{1}, z_{2}\right) \quad \text { if }\left|z_{1}\right| \leq r_{1},\left|z_{2}\right| \leq r_{2} \quad \text { and } \\
& f_{s, r}\left(z_{1}, z_{2}\right)=f\left(z_{1}, z_{2}\right) \quad \text { if }\left(z_{1}, z_{2}\right) \in\left(r_{1}, \infty\right) \times\left(r_{2}, \infty\right) .
\end{aligned}
$$

From the hypothesis on $f$, it is clear that each $f_{s, r}$ is bounded on $[0, \infty) \times[0, \infty)$, which implies that

$$
\left|R_{n, m}^{q_{1}, q_{2}}\left(f_{s, r}\right)\left(z_{1}, z_{2}\right)\right| \leq \sum_{k=0}^{n} \sum_{j=0}^{m}\left|p_{n, k}\left(z_{1}\right)\right|\left|p_{m, j}\left(z_{2}\right)\right| M_{f_{s, r}}<\infty,
$$

where $M_{f_{s, r}}$ is a constant depending on $f_{s, r}$, so all $R_{n, m}^{q_{1}, q_{2}}\left(f_{s, r}\right)$ are well defined for all $n, m \in \mathbb{N}$, $n \geq n_{0}, m \geq m_{0}, r_{1}<\frac{\left[n_{0}\right]_{q}^{1-\beta}}{2}, r_{2}<\frac{\left[m_{0}\right]_{q}^{1-\beta}}{2}$ and $\left(z_{1}, z_{2}\right) \in D_{r_{1}} \times D_{r_{2}}$.

Defining

$$
\begin{aligned}
& f_{s, r, k, j}\left(z_{1}, z_{2}\right)=c_{k, j} e_{k, j}\left(z_{1}, z_{2}\right) \quad \text { if }\left|z_{1}\right| \leq r_{1},\left|z_{2}\right| \leq r_{2} \quad \text { and } \\
& f_{s, r, k, j}\left(z_{1}, z_{2}\right)=\frac{f\left(z_{1}, z_{2}\right)}{(s+1)(r+1)} \quad \text { if }\left(z_{1}, z_{2}\right) \in\left(r_{1}, \infty\right) \times\left(r_{2}, \infty\right)
\end{aligned}
$$

It is clear that each $f_{s, r, k, j}$ is bounded on $[0, \infty) \times[0, \infty)$ and

$$
f_{s, r}\left(z_{1}, z_{2}\right)=\sum_{k=0}^{s} \sum_{j=0}^{r} f_{s, r, k, j}\left(z_{1}, z_{2}\right) .
$$

From the linearity of $R_{n, m}^{q_{1}, q_{2}}$, we have

$$
R_{n, m}^{q_{1}, q_{2}}\left(f_{s, r}\right)\left(z_{1}, z_{2}\right)=\sum_{k=0}^{s} \sum_{j=0}^{r} c_{k, j} R_{n, m}^{q_{1}, q_{2}}\left(e_{k, j}\right)\left(z_{1}, z_{2}\right) .
$$

It suffices to prove that

$$
\lim _{s, r \rightarrow \infty} R_{n, m}^{q_{1}, q_{2}}\left(f_{s, r}\right)\left(z_{1}, z_{2}\right)=R_{n, m}^{q_{1}, q_{2}}(f)\left(z_{1}, z_{2}\right)
$$

for any fixed $n, m \in \mathbb{N}, n \geq n_{0}, m \geq m_{0},\left|z_{1}\right| \leq r_{1}$ and $\left|z_{2}\right| \leq r_{2}$. Since

$$
\left\|f_{s, r}-f\right\|_{B([0, \infty) \times[0, \infty))} \leq\left\|f_{s, r}-f\right\|_{r_{1}, r_{2}},
$$


we can write

$$
\begin{aligned}
\left|R_{n, m}^{q_{1}, q_{2}}\left(f_{s, r}\right)\left(z_{1}, z_{2}\right)-R_{n, m}^{q_{1}, q_{2}}(f)\left(z_{1}, z_{2}\right)\right| & \leq M_{r_{1}, r_{2}, m, n}^{q_{1}, q_{2}}\left\|f_{s, r}-f\right\|_{B([0, \infty) \times[0, \infty))} \\
& \leq M_{r_{1}, r_{2}, m, n}^{q_{1}, q_{2}}\left\|f_{s, r}-f\right\|_{r_{1}, r_{2}}
\end{aligned}
$$

for $\left|z_{1}\right| \leq r_{1}$ and $\left|z_{2}\right| \leq r_{2}$

In equation (3), taking the limit as $s, r \rightarrow \infty$ and using $\lim _{s, r \rightarrow \infty}\left\|f_{s, r}-f\right\|_{r_{1}, r_{2}}=0$, we get the result.

Lemma 2 Let $n_{0}, m_{0} \geq 2,0<\beta \leq \frac{2}{3}, \frac{1}{2}<r_{1}<R_{1} \leq \frac{\left[n_{0}\right]_{q_{1}}^{1-\beta}}{2}$ and $\frac{1}{2}<r_{2}<R_{2} \leq \frac{\left[m_{0}\right]_{q_{2}}^{1-\beta}}{2}$. For all $n \geq n_{0}, m \geq m_{0},\left|z_{1}\right| \leq r_{1},\left|z_{2}\right| \leq r_{2}$ and $k=0,1,2, \ldots$ the following inequality holds:

$$
\left|R_{n, m}^{q_{1}, q_{2}}\left(e_{k, j}\right)\left(z_{1}, z_{2}\right)\right| \leq k ! j !\left(20 r_{1}\right)^{k}\left(20 r_{2}\right)^{j}
$$

Proof Using Lemma 4 in [19], the lemma is easily proved, so we omit the proof of the lemma.

\section{Main results}

Let us denote by $A_{C}$ the space of all uniformly continuous complex valued functions defined on $\left(D_{R_{1}} \cup\left[R_{1}, \infty\right)\right) \times\left(D_{R_{2}} \cup\left[R_{2}, \infty\right)\right)$, bounded on $[0, \infty) \times[0, \infty)$ and analytic in $D_{R_{1}} \times D_{R_{2}}$ and for which there exist $M>0,0<A_{1}<\frac{1}{20 r_{1}}$ and $0<A_{2}<\frac{1}{20 r_{2}}$ with $\left|c_{k, j}\right| \leq M \frac{A_{1}^{k} A_{2}^{j}}{k ! j !}$ for all $k, j=0,1,2, \ldots$ (which implies $\left|f\left(z_{1}, z_{2}\right)\right| \leq M e^{A_{1}\left|z_{1}\right|+A_{2}\left|z_{2}\right|}$ for all $\left.\left(z_{1}, z_{2}\right) \in D_{R_{1}} \times D_{R_{2}}\right)$.

We have the following upper estimate.

Theorem 1 Let $q_{1}=\left(q_{1, n}\right)$ and $q_{2}=\left(q_{2, m}\right)$ be sequences satisfying the conditions given in equation (2) and let $n_{0}, m_{0} \geq 2,0<\beta \leq \frac{2}{3}, \frac{1}{2}<r_{1}<R_{1} \leq \frac{\left[n_{0}\right]_{q_{1}}^{1-\beta}}{2}$ and $\frac{1}{2}<r_{2}<R_{2} \leq \frac{\left[m_{0}\right]_{q_{2}}^{1-\beta}}{2}$. Iff $\in A_{C}$, then for all $n \geq n_{0}, m \geq m_{0},\left|z_{1}\right| \leq r_{1}$ and $\left|z_{2}\right| \leq r_{2}$ the following inequality holds:

$$
\left|R_{n, m}^{q_{1}, q_{2}}(f)\left(z_{1}, z_{2}\right)-f\left(z_{1}, z_{2}\right)\right| \leq\left(a_{n}+\frac{1}{b_{n}}\right) C^{3}(f)+\left(a_{m}+\frac{1}{b_{m}}\right) C^{4}(f)
$$

where

$$
\begin{aligned}
& C^{3}(f)=\max \left\{M r_{1} r_{2} e^{2 r_{1} A_{1}+r_{2} A_{2}}, 9 M e^{r_{2} A_{2}} \sum_{k=1}^{\infty}(k-1)\left(20 r_{1} A_{1}\right)^{k-1}\right\}, \\
& C^{4}(f)=\max \left\{\begin{array}{c}
2 M\left(r_{2}\right)^{2} e^{2 r_{2} A_{2}} \sum_{k=0}^{\infty}\left(20 r_{1} A_{1}\right)^{k}, \\
9 M \sum_{k=0}^{\infty}\left(20 r_{1} A_{1}\right)^{k} \sum_{j=1}^{\infty}(j-1)\left(20 r_{2} A_{2}\right)^{j-1}
\end{array}\right\},
\end{aligned}
$$

and also the series $\sum_{k=0}^{\infty}\left(20 r_{1} A_{1}\right)^{k}, \sum_{k=1}^{\infty}(k-1)\left(20 r_{1} A_{1}\right)^{k-1}$ and $\sum_{j=1}^{\infty}(j-1)\left(20 r_{2} A_{2}\right)^{j-1}$ are convergent.

Proof Using Lemma 1, we can write

$$
\left|R_{n, m}^{q_{1}, q_{2}}(f)\left(z_{1}, z_{2}\right)-f\left(z_{1}, z_{2}\right)\right| \leq \sum_{k=0}^{\infty} \sum_{j=0}^{\infty}\left|c_{k, j}\right|\left|R_{n, m}^{q_{1}, q_{2}}\left(e_{k, j}\right)\left(z_{1}, z_{2}\right)-e_{k, j}\left(z_{1}, z_{2}\right)\right| .
$$


Taking into account Lemma 4 in [19] and the estimate given in the proof of Theorem 2 in [19], for all $\left|z_{1}\right| \leq r_{1}$ and $\left|z_{2}\right| \leq r_{2}$, we obtain

$$
\begin{aligned}
& \left|R_{n, m}^{q_{1}, q_{2}}\left(e_{k, j}\right)\left(z_{1}, z_{2}\right)-e_{k, j}\left(z_{1}, z_{2}\right)\right| \\
& =\left|R_{n}^{q_{1}}\left(e_{1}^{k}\right)\left(z_{1}\right) \cdot R_{m}^{q_{2}}\left(e_{2}^{j}\right)\left(z_{2}\right)-z_{1}^{k} z_{2}^{j}\right| \\
& \leq\left|R_{n}^{q_{1}}\left(e_{1}^{k}\right)\left(z_{1}\right)\right|\left|R_{m}^{q_{2}}\left(e_{2}^{j}\right)\left(z_{2}\right)-z_{2}^{j}\right|+\left|z_{2}^{j}\right|\left|R_{n}^{q_{1}}\left(e_{1}^{k}\right)\left(z_{1}\right)-z_{1}^{k}\right| \\
& \leq(k !)\left(20 r_{1}\right)^{k}\left\{2 a_{m}\left(r_{2}\right)^{2} j\left(2 r_{2}\right)^{j-1}+\frac{9}{b_{m}}(j-1)(j !)\left(20 r_{2}\right)^{j-1}\right\} \\
& \quad+\left(r_{2}\right)^{j}\left\{2 a_{n}\left(r_{1}\right)^{2} k\left(2 r_{1}\right)^{k-1}+\frac{9}{b_{n}}(k-1)(k !)\left(20 r_{1}\right)^{k-1}\right\} \\
& =2 a_{n}\left(r_{1}\right)^{2}\left(2 r_{1}\right)^{k-1} j\left(r_{2}\right)^{j}+2 a_{m}\left(r_{2}\right)^{2}(k !)\left(20 r_{1}\right)^{k}\left(2 r_{2}\right)^{j-1} \\
& \quad+\frac{9}{b_{n}}(k-1)(k !)\left(20 r_{1}\right)^{k-1}\left(r_{2}\right)^{j}+\frac{9}{b_{m}}(k !)\left(20 r_{1}\right)^{k}(j-1)(j !)\left(20 r_{2}\right)^{j-1} .
\end{aligned}
$$

Applying equation (5) in equation (4), we get

$$
\begin{aligned}
& \left|R_{n, m}^{q_{1}, q_{2}}(f)\left(z_{1}, z_{2}\right)-f\left(z_{1}, z_{2}\right)\right| \\
& \leq a_{n} M r_{1} r_{2} e^{2 r_{1} A_{1}+r_{2} A_{2}}+2 a_{m} M\left(r_{2}\right)^{2} e^{2 r_{2} A_{2}} \sum_{k=0}^{\infty}\left(20 r_{1} A_{1}\right)^{k} \\
& \quad+\frac{9 M}{b_{n}} e^{r_{2} A_{2}} \sum_{k=1}^{\infty}(k-1)\left(20 r_{1} A_{1}\right)^{k-1}+\frac{9 M}{b_{m}} \sum_{k=0}^{\infty}\left(20 r_{1} A_{1}\right)^{k} \sum_{j=1}^{\infty}(j-1)\left(20 r_{2} A_{2}\right)^{j-1} .
\end{aligned}
$$

Choosing $C^{3}(f)$ and $C^{4}(f)$ as given in the theorem, we reach the desired result.

For $f\left(z_{1}, z_{2}\right)$, we define the parametric extensions of the Voronovskaja formula by

$$
\begin{aligned}
z_{1} L_{n, q_{1}}(f)\left(z_{1}, z_{2}\right):= & R_{n}^{q_{1}}\left(f\left(\cdot, z_{2}\right)\right)\left(z_{1}\right)-f\left(z_{1}, z_{2}\right)-\psi_{n, q_{1}}^{1}\left(z_{1}\right) \frac{\partial f}{\partial z_{1}}\left(z_{1}, z_{2}\right) \\
& -\frac{1}{2} \psi_{n, q_{1}}^{2}\left(z_{1}\right) \frac{\partial^{2} f}{\partial z_{1}^{2}}\left(z_{1}, z_{2}\right)
\end{aligned}
$$

and

$$
\begin{aligned}
z_{2} L_{m, q_{2}}(f)\left(z_{1}, z_{2}\right):= & R_{m}^{q_{2}}\left(f\left(z_{1}, \cdot\right)\right)\left(z_{2}\right)-f\left(z_{1}, z_{2}\right)-\psi_{m, q_{2}}^{1}\left(z_{2}\right) \frac{\partial f}{\partial z_{2}}\left(z_{1}, z_{2}\right) \\
& -\frac{1}{2} \psi_{m, q_{2}}^{2}\left(z_{2}\right) \frac{\partial^{2} f}{\partial z_{2}^{2}}\left(z_{1}, z_{2}\right)
\end{aligned}
$$

where $\psi_{k, q}^{i}(z)=R_{k}^{q}\left((t-z)^{i} ; z\right)$ for $i=1,2$ given in Lemma 6 in [19].

Their product (composition) gives

$$
\begin{aligned}
& z_{2} L_{m, q_{2}}(f)\left(z_{1}, z_{2}\right) \circ z_{1} L_{n, q_{1}}(f)\left(z_{1}, z_{2}\right) \\
& \quad=R_{m}^{q_{2}}\left(R_{n}^{q_{1}}(f(\cdot, \cdot))\left(z_{1}\right)-f\left(z_{1}, \cdot\right)-\psi_{n, q_{1}}^{1}\left(z_{1}\right) \frac{\partial f}{\partial z_{1}}\left(z_{1}, \cdot\right)-\psi_{n, q_{1}}^{2}\left(z_{1}\right) \frac{\partial^{2} f}{\partial z_{1}^{2}}\left(z_{1}, \cdot\right)\right)\left(z_{2}\right)
\end{aligned}
$$




$$
\begin{aligned}
& -\left[R_{n}^{q_{1}}\left(f\left(\cdot, z_{2}\right)\right)\left(z_{1}\right)-f\left(z_{1}, z_{2}\right)-\psi_{n, q_{1}}^{1}\left(z_{1}\right) \frac{\partial f}{\partial z_{1}}\left(z_{1}, z_{2}\right)-\psi_{n, q_{1}}^{2}\left(z_{1}\right) \frac{\partial^{2} f}{\partial z_{1}^{2}}\left(z_{1}, z_{2}\right)\right] \\
& -\psi_{m, q_{2}}^{1}\left(z_{2}\right)\left[R_{n}^{q_{1}}\left(\frac{\partial f}{\partial z_{2}}\left(\cdot, z_{2}\right)\right)\left(z_{1}\right)-\frac{\partial f}{\partial z_{2}}\left(z_{1}, z_{2}\right)-\psi_{n, q_{1}}^{1}\left(z_{1}\right) \frac{\partial^{2} f}{\partial z_{2} \partial z_{1}}\left(z_{1}, z_{2}\right)\right. \\
& \left.-\psi_{n, q_{1}}^{2}\left(z_{1}\right) \frac{\partial^{3} f}{\partial z_{2} \partial z_{1}^{2}}\left(z_{1}, z_{2}\right)\right] \\
& -\psi_{m, q_{2}}^{2}\left(z_{2}\right)\left[R_{n}^{q_{1}}\left(\frac{\partial^{2} f}{\partial z_{2}^{2}}\left(\cdot, z_{2}\right)\right)\left(z_{1}\right)-\frac{\partial^{2} f}{\partial z_{2}^{2}}\left(z_{1}, z_{2}\right)-\psi_{n, q_{1}}^{1}\left(z_{1}\right) \frac{\partial^{3} f}{\partial z_{2}^{2} \partial z_{1}}\left(z_{1}, z_{2}\right)\right. \\
& \left.-\psi_{n, q_{1}}^{2}\left(z_{1}\right) \frac{\partial^{4} f}{\partial z_{2}^{2} \partial z_{1}^{2}}\left(z_{1}, z_{2}\right)\right]:=E_{1}-E_{2}+E_{3}-E_{4} .
\end{aligned}
$$

After a simple calculation, we obtain the commutativity property,

$$
z_{2} L_{m, q_{2}}(f)\left(z_{1}, z_{2}\right) \circ z_{1} L_{n, q_{1}}(f)\left(z_{1}, z_{2}\right)=z_{1} L_{n, q_{1}}(f)\left(z_{1}, z_{2}\right) \circ z_{2} L_{m, q_{2}}(f)\left(z_{1}, z_{2}\right) .
$$

In the following a Voronovskaja-type result for the operators (1) is presented. It will be the product of the parametric extensions generated by Voronovskaja's formula in the univariate case.

Theorem 2 Let $q_{1}=\left(q_{1, n}\right)$ and $q_{2}=\left(q_{2, m}\right)$ be sequences satisfying the conditions given in equation (2) and let $n_{0}, m_{0} \geq 2,0<\beta \leq \frac{2}{3}, \frac{1}{2}<r_{1}<R_{1} \leq \frac{\left[n_{0}\right]_{q_{1}}^{1-\beta}}{2}$ and $\frac{1}{2}<r_{2}<R_{2} \leq \frac{\left[m_{0}\right]_{q_{2}}^{1-\beta}}{2}$. If $f \in A_{C}$, then for all $n \geq n_{0}, m \geq m_{0},\left|z_{1}\right| \leq r_{1}$ and $\left|z_{2}\right| \leq r_{2}$ the following inequality holds:

$$
\left|z_{2} L_{m, q_{2}}(f)\left(z_{1}, z_{2}\right) \circ z_{1} L_{n, q_{1}}(f)\left(z_{1}, z_{2}\right)\right| \leq C^{5}(f)\left[\left(a_{n}+\frac{1}{b_{n}}\right)^{2}+\left(a_{m}+\frac{1}{b_{m}}\right)^{2}\right],
$$

where $C^{5}(f)=\frac{1}{2} \max \left\{C_{r_{1}, r_{2}}^{1}(f), C_{r_{1}, r_{2}}^{2}(f)\right\}, C^{\prime}$, and $C^{\prime \prime}$ are fixed constants,

$$
\begin{aligned}
C_{r_{1}, r_{2}}^{1}(f)= & M r_{1}^{3} e^{r_{2} A_{2}} \sum_{k=2}^{\infty}(k-2)(k-1) k(k+1)\left(20 r_{1} A_{1}\right)^{k-3} \\
& \times \max \left\{C^{\prime} e^{-r_{2} A_{2}} \sum_{j=0}^{\infty}\left(20 r_{2} A_{2}\right)^{j}, C^{\prime}, C^{\prime} r_{2} A_{2}, C^{\prime \prime}\left(1+r_{2}+r_{2}^{2}\right) r_{2} A_{2}^{2}\right\}
\end{aligned}
$$

and

$$
\begin{aligned}
C_{r_{1}, r_{2}}^{2}(f)= & M r_{2}^{3} e^{r_{1} A_{1}} \sum_{j=2}^{\infty}(j-2)(j-1) j(j+1)\left(20 r_{2} A_{2}\right)^{j-3} \\
& \times \max \left\{C^{\prime} e^{-r_{1} A_{1}} \sum_{k=0}^{\infty}\left(20 r_{1} A_{1}\right)^{k}, C^{\prime}, C^{\prime} r_{1} A_{1}, C^{\prime \prime}\left(1+r_{1}+r_{1}^{2}\right) r_{1} A_{1}^{2}\right\} .
\end{aligned}
$$

Proof From the analyticity of $f$ in $D_{R_{1}} \times D_{R_{2}}$, since all partial derivatives of $f$ are analytic in $D_{R_{1}} \times D_{R_{2}}$, using Lemma 1 , we can write

$$
\begin{aligned}
& R_{n}^{q_{1}}\left(f\left(\cdot, z_{2}\right)\right)\left(z_{1}\right)-f\left(z_{1}, z_{2}\right)-\psi_{n, q_{1}}^{1}\left(z_{1}\right) \frac{\partial f}{\partial z_{1}}\left(z_{1}, z_{2}\right)-\psi_{n, q_{1}}^{2}\left(z_{1}\right) \frac{\partial^{2} f}{\partial z_{1}^{2}}\left(z_{1}, z_{2}\right) \\
& \quad=\sum_{k=2}^{\infty} f_{k}\left(z_{2}\right)\left[R_{n}^{q_{1}}\left(e_{1}^{k}\right)\left(z_{1}\right)-e_{1}^{k}\left(z_{1}\right)-\psi_{n, q_{1}}^{1}\left(z_{1}\right) k z_{1}^{k-1}-\psi_{n, q_{1}}^{2}\left(z_{1}\right) k(k-1) z_{1}^{k-2}\right] .
\end{aligned}
$$


Applying now $R_{m}^{q_{2}}$ to equation (7) with respect to $z_{2}$ and Lemma 1 in [19], we obtain

$$
\begin{aligned}
E_{1}= & \sum_{k=2}^{\infty} R_{m}^{q_{2}}\left(f_{k}\right)\left(z_{2}\right)\left[R_{n}^{q_{1}}\left(e_{1}^{k}\right)\left(z_{1}\right)-e_{1}^{k}\left(z_{1}\right)-\psi_{n, q_{1}}^{1}\left(z_{1}\right) k z_{1}^{k-1}\right. \\
& \left.-\psi_{n, q_{1}}^{2}\left(z_{1}\right) k(k-1) z_{1}^{k-2}\right] \\
= & \sum_{k=2}^{\infty} \sum_{j=0}^{\infty} c_{k, j} R_{m}^{q_{2}}\left(e_{2}^{j}\right)\left(z_{2}\right)\left[R_{n}^{q_{1}}\left(e_{1}^{k}\right)\left(z_{1}\right)-e_{1}^{k}\left(z_{1}\right)-\psi_{n, q_{1}}^{1}\left(z_{1}\right) k z_{1}^{k-1}\right. \\
& \left.-\psi_{n, q_{1}}^{2}\left(z_{1}\right) k(k-1) z_{1}^{k-2}\right] .
\end{aligned}
$$

In equation (8), passing now to absolute value for $\left|z_{1}\right| \leq r_{1}$ and $\left|z_{2}\right| \leq r_{2}$ and taking into account the Lemma 4 in [19] and the estimate given in the proof of Theorem 3 in [19], it follows that

$$
\begin{aligned}
\left|E_{1}\right| & \leq\left(a_{n}+\frac{1}{b_{n}}\right)^{2} \sum_{j=0}^{\infty}\left|c_{k, j}\right| j !\left(20 r_{2}\right)^{j} \sum_{k=2}^{\infty} C(k-2)(k-1) k(k+1) k !\left(20 r_{1}\right)^{k+3} \\
& \leq\left(a_{n}+\frac{1}{b_{n}}\right)^{2} M C^{\prime} r_{1}^{3} \sum_{j=0}^{\infty}\left(20 r_{2} A_{2}\right)^{j} \sum_{k=2}^{\infty}(k-2)(k-1) k(k+1)\left(20 r_{1} A_{1}\right)^{k-3}
\end{aligned}
$$

for $\left|z_{1}\right| \leq r_{1}$ and $\left|z_{2}\right| \leq r_{2}$.

Similarly, using the estimate given in the proof of Theorem 3 in [19] for $\left|z_{1}\right| \leq r_{1}$ and $\left|z_{2}\right| \leq r_{2}$ we have

$$
\begin{aligned}
\left|E_{2}\right| & \leq \sum_{k=2}^{\infty}\left|f_{k}\left(z_{2}\right)\right|\left|R_{n}^{q_{1}}\left(e_{1}^{k}\right)\left(z_{1}\right)-e_{1}^{k}\left(z_{1}\right)-\psi_{n, q_{1}}^{1}\left(z_{1}\right) k z_{1}^{k-1}-\psi_{n, q_{1}}^{2}\left(z_{1}\right) k(k-1) z_{1}^{k-2}\right| \\
& \leq\left(a_{n}+\frac{1}{b_{n}}\right)^{2} \sum_{j=0}^{\infty}\left|c_{k, j}\right| r_{2}^{j} \sum_{k=2}^{\infty} C(k-2)(k-1) k(k+1) k !\left(20 r_{1}\right)^{k+3} \\
& \leq\left(a_{n}+\frac{1}{b_{n}}\right)^{2} M C^{\prime} r_{1}^{3} e^{r_{2} A_{2}} \sum_{k=2}^{\infty}(k-2)(k-1) k(k+1)\left(20 r_{1} A_{1}\right)^{k-3} .
\end{aligned}
$$

Using

$$
\begin{aligned}
R_{n}^{q_{1}}\left(\frac{\partial f}{\partial z_{2}}\left(\cdot, z_{2}\right)\right)\left(z_{1}\right) & =\sum_{k=0}^{\infty} \frac{\partial f_{k}}{\partial z_{2}}\left(z_{2}\right) R_{n}^{q_{1}}\left(e_{1}^{k}\right)\left(z_{1}\right) \\
& =\sum_{k=0}^{\infty} \sum_{j=1}^{\infty} c_{k, j} j z_{2}^{j-1} R_{n}^{q_{1}}\left(e_{1}^{k}\right)\left(z_{1}\right),
\end{aligned}
$$

we can write

$$
\begin{aligned}
E_{3}= & \psi_{m, q_{2}}^{1}\left(z_{2}\right)\left[R_{n}^{q_{1}}\left(\frac{\partial f}{\partial z_{2}}\left(\cdot, z_{2}\right)\right)\left(z_{1}\right)-\frac{\partial f}{\partial z_{2}}\left(z_{1}, z_{2}\right)-\psi_{n, q_{1}}^{1}\left(z_{1}\right) \frac{\partial^{2} f}{\partial z_{2} \partial z_{1}}\left(z_{1}, z_{2}\right)\right. \\
& \left.-\psi_{n, q_{1}}^{2}\left(z_{1}\right) \frac{\partial^{3} f}{\partial z_{2} \partial z_{1}^{2}}\left(z_{1}, z_{2}\right)\right]
\end{aligned}
$$




$$
\begin{aligned}
= & \psi_{m, q_{2}}^{1}\left(z_{2}\right) \sum_{k=2}^{\infty} \sum_{j=1}^{\infty} c_{k, j} j z_{2}^{j-1}\left[R_{n}^{q_{1}}\left(e_{1}^{k}\right)\left(z_{1}\right)-e_{1}^{k}\left(z_{1}\right)-\psi_{n, q_{1}}^{1}\left(z_{1}\right) k z_{1}^{k-1}\right. \\
& \left.-\psi_{n, q_{1}}^{2}\left(z_{1}\right) k(k-1) z_{1}^{k-2}\right] .
\end{aligned}
$$

Considering Lemma 6 in [19] and the estimate given in the proof of Theorem 3 in [19], for $\left|z_{1}\right| \leq r_{1}$ and $\left|z_{2}\right| \leq r_{2}$, we obtain

$$
\begin{aligned}
\left|E_{3}\right| & \leq\left(a_{n}+\frac{1}{b_{n}}\right)^{2}\left|\psi_{m, q_{2}}^{1}\left(z_{2}\right)\right| \sum_{k=2}^{\infty} \sum_{j=1}^{\infty}\left|c_{k, j}\right| j r_{2}^{j-1} C(k-2)(k-1) k(k+1) k !\left(20 r_{1}\right)^{k+3} \\
& \leq\left(a_{n}+\frac{1}{b_{n}}\right)^{2} M C^{\prime} r_{1}^{3} r_{2} A_{2} e^{r_{2} A_{2}} \sum_{k=2}^{\infty}(k-2)(k-1) k(k+1)\left(20 r_{1} A_{1}\right)^{k-3}
\end{aligned}
$$

and also, using

$$
\begin{aligned}
R_{n}\left(\frac{\partial^{2} f}{\partial z_{2}^{2}}\left(\cdot, z_{2}\right)\right)\left(z_{1}\right) & =\sum_{k=0}^{\infty} \frac{\partial^{2} f_{k}}{\partial z_{2}^{2}}\left(z_{2}\right) R_{n}\left(e_{1}^{k}\right)\left(z_{1}\right) \\
& =\sum_{k=0}^{\infty} \sum_{j=2}^{\infty} c_{k, j} j(j-1) z_{2}^{j-2} R_{n}\left(e_{1}^{k}\right)\left(z_{1}\right),
\end{aligned}
$$

we can write

$$
\begin{aligned}
E_{4}= & \psi_{m, q_{2}}^{2}\left(z_{2}\right)\left[R_{n}\left(\frac{\partial^{2} f}{\partial z_{2}^{2}}\left(\cdot, z_{2}\right)\right)\left(z_{1}\right)-\frac{\partial^{2} f}{\partial z_{2}^{2}}\left(z_{1}, z_{2}\right)-\psi_{n, q_{1}}^{1}\left(z_{1}\right) \frac{\partial^{3} f}{\partial z_{1} \partial z_{2}^{2}}\left(z_{1}, z_{2}\right)\right. \\
& \left.-\psi_{n, q_{1}}^{2}\left(z_{1}\right) \frac{\partial^{4} f}{\partial z_{1}^{2} \partial z_{2}^{2}}\left(z_{1}, z_{2}\right)\right] \\
= & \psi_{m, q_{2}}^{2}\left(z_{2}\right) \sum_{k=2}^{\infty} \sum_{j=2}^{\infty} c_{k, j}(j-1) z_{2}^{j-2}\left[R_{n}^{q_{1}}\left(e_{1}^{k}\right)\left(z_{1}\right)-e_{1}^{k}\left(z_{1}\right)-\psi_{n, q_{1}}^{1}\left(z_{1}\right) k z_{1}^{k-1}\right. \\
& \left.-\psi_{n, q_{1}}^{2}\left(z_{1}\right) k(k-1) z_{1}^{k-2}\right] .
\end{aligned}
$$

Taking into account Lemma 6 in [19] and the estimate given in the proof of Theorem 3 in [19], for $\left|z_{1}\right| \leq r_{1}$ and $\left|z_{2}\right| \leq r_{2}$ we get

$$
\begin{aligned}
\left|E_{4}\right| \leq & \left(a_{n}+\frac{1}{b_{n}}\right)^{2}\left|\psi_{m, q_{2}}^{2}\left(z_{2}\right)\right| \sum_{j=2}^{\infty} j(j-1) r_{2}^{j-2} \\
& \times \sum_{k=2}^{\infty}\left|c_{k, j}\right| C(k-2)(k-1) k(k+1) k !\left(20 r_{1}\right)^{k+3} \\
\leq & \left(a_{n}+\frac{1}{b_{n}}\right)^{2} M C^{\prime \prime} r_{1}^{3}\left(1+r_{2}+r_{2}^{2}\right) r_{2} A_{2}^{2} e^{A_{2} r_{2}} \\
& \times \sum_{k=2}^{\infty}(k-2)(k-1) k(k+1)\left(20 A_{1} r_{1}\right)^{k-3}
\end{aligned}
$$


for $\left|z_{1}\right| \leq r_{1}$ and $\left|z_{2}\right| \leq r_{2}$. Using equations (9)-(12), we get

$$
\begin{aligned}
\left|z_{2} L_{m, q_{2}}(f)\left(z_{1}, z_{2}\right) \circ z_{1} L_{n, q_{1}}(f)\left(z_{1}, z_{2}\right)\right| & \leq\left|E_{1}\right|+\left|E_{2}\right|+\left|E_{3}\right|+\left|E_{4}\right| \\
& \leq C_{r_{1}, r_{2}}^{1}(f)\left(a_{n}+\frac{1}{b_{n}}\right)^{2} .
\end{aligned}
$$

If we estimate $\left|z_{1} L_{n, q_{1}}(f)\left(z_{1}, z_{2}\right) \circ z_{2} L_{m, q_{2}}(f)\left(z_{1}, z_{2}\right)\right|$, then by reason of the symmetry we get a similar order of approximation, simply interchanging above the places of $n$ with $m$ and $r_{1}$ with $r_{2}$.

In conclusion, using the commutativity property, we reach the result.

Let us denote by $A_{C}^{(2)}$ the space of all complex valued functions where they and their first and second partial derivatives are uniformly continuous on $\left(D_{R_{1}} \cup\left[R_{1}, \infty\right)\right) \times\left(D_{R_{2}} \cup\right.$ $\left[R_{2}, \infty\right)$ ), bounded on $[0, \infty) \times[0, \infty)$ and analytic in $D_{R_{1}} \times D_{R_{2}}$, and there exist $M>0$, $0<A_{1}<\frac{1}{20 r_{1}}, 0<A_{2}<\frac{1}{20 r_{2}}$ with $\left|c_{k, j}\right| \leq M \frac{A_{1}^{k} A_{2}^{j}}{k ! j !}$ (which implies $\left|f\left(z_{1}, z_{2}\right)\right| \leq M e^{A_{1}\left|z_{1}\right|+A_{2}\left|z_{2}\right|}$ for all $\left.\left(z_{1}, z_{2}\right) \in D_{R_{1}} \times D_{R_{2}}\right)$.

Theorems 1 and 2 will be used to find the exact degree in the approximation of $R_{n, n}^{q_{1}, q_{2}}(f)$. In this sense, we have the following lower estimate.

Theorem 3 Let $q_{1}=\left(q_{1, n}\right)$ and $q_{2}=\left(q_{2, n}\right)$ be sequences satisfying the conditions given in equation (2) and let $n_{0} \geq 2,0<\beta<\frac{1}{2}, \frac{1}{2}<r_{1}<R_{1} \leq \frac{\left[n_{0}\right]_{q_{1}}^{1-\beta}}{2}$ and $\frac{1}{2}<r_{2}<R_{2} \leq \frac{\left[n_{0}\right]_{q_{2}}^{1-\beta}}{2}$. If $f \in A_{C}^{(2)}$ and $f$ is not a solution of the complex partial differential equation

$$
K(f)\left(z_{1}, z_{2}\right)=z_{1} \frac{\partial^{2} f}{\partial z_{1}^{2}}\left(z_{1}, z_{2}\right)+z_{2} \frac{\partial^{2} f}{\partial z_{2}^{2}}\left(z_{1}, z_{2}\right)=0,
$$

then for all $n \geq n_{0}$ we have

$$
\left\|R_{n, n}^{q_{1}, q_{2}}(f)-f\right\|_{r_{1}, r_{2}} \geq \frac{1}{36\left(1+a_{n} b_{n}\right)}\left(a_{n}+\frac{1}{b_{n}}\right)\|K(f)\|_{r_{1}, r_{2}} .
$$

Proof From equation (6), we can write

$$
\begin{aligned}
& R_{n, n}^{q_{1}, q_{2}}(f)\left(z_{1}, z_{2}\right)-f\left(z_{1}, z_{2}\right) \\
& =2\left(a_{n}+\frac{1}{b_{n}}\right)\left\{K_{n}(f)\left(z_{1}, z_{2}\right)+2\left(a_{n}+\frac{1}{b_{n}}\right)\left[\frac{D_{n}(f)\left(z_{1}, z_{2}\right)}{4\left(a_{n}+\frac{1}{b_{n}}\right)^{2}}\right]\right. \\
& \left.\quad+E_{n}(f)\left(z_{1}, z_{2}\right)+F_{n}(f)\left(z_{1}, z_{2}\right)+G_{n}(f)\left(z_{1}, z_{2}\right)\right\},
\end{aligned}
$$

where

$$
\begin{aligned}
& D_{n}(f)\left(z_{1}, z_{2}\right)=z_{2} L_{n, q_{2}}(f)\left(z_{1}, z_{2}\right) \circ z_{1} L_{n, q_{1}}(f)\left(z_{1}, z_{2}\right), \\
& E_{n}(f)\left(z_{1}, z_{2}\right)=\frac{z_{1} L_{n, q_{1}}(f)\left(z_{1}, z_{2}\right)+z_{2} L_{n, q_{2}}(f)\left(z_{1}, z_{2}\right)}{2\left(a_{n}+\frac{1}{b_{n}}\right)}, \\
& F_{n}(f)\left(z_{1}, z_{2}\right)=\sum_{h=1}^{4} F_{n}^{h}(f)\left(z_{1}, z_{2}\right)
\end{aligned}
$$


with

$$
\begin{aligned}
F_{n}^{1}(f)\left(z_{1}, z_{2}\right)= & \frac{b_{n} \psi_{n, q_{1}}^{1}\left(z_{1}\right)}{2\left(1+a_{n} b_{n}\right)}\left[R_{n}^{q_{2}}\left(\frac{\partial f}{\partial z_{1}}\left(z_{1}, \cdot\right)\right)\left(z_{2}\right)-\frac{\partial f}{\partial z_{1}}\left(z_{1}, z_{2}\right)\right], \\
F_{n}^{2}(f)\left(z_{1}, z_{2}\right)= & \frac{b_{n} \psi_{n, q_{2}}^{1}\left(z_{2}\right)}{2\left(1+a_{n} b_{n}\right)}\left[R_{n}^{q_{1}}\left(\frac{\partial f}{\partial z_{2}}\left(\cdot, z_{2}\right)\right)\left(z_{1}\right)-\frac{\partial f}{\partial z_{2}}\left(z_{1}, z_{2}\right)\right], \\
F_{n}^{3}(f)\left(z_{1}, z_{2}\right)= & \frac{b_{n} \psi_{n, q_{1}}^{2}\left(z_{1}\right)}{4\left(1+a_{n} b_{n}\right)}\left[R_{n}^{q_{2}}\left(\frac{\partial^{2} f}{\partial z_{1}^{2}}\left(z_{1}, \cdot\right)\right)\left(z_{2}\right)-\frac{\partial^{2} f}{\partial z_{1}^{2}}\left(z_{1}, z_{2}\right)\right], \\
F_{n}^{4}(f)\left(z_{1}, z_{2}\right)= & \frac{b_{n} \psi_{n, q_{2}}^{2}\left(z_{2}\right)}{4\left(1+a_{n} b_{n}\right)}\left[R_{n}^{q_{1}}\left(\frac{\partial^{2} f}{\partial z_{2}^{2}}\left(\cdot, z_{2}\right)\right)\left(z_{1}\right)-\frac{\partial^{2} f}{\partial z_{2}^{2}}\left(z_{1}, z_{2}\right)\right], \\
G_{n}(f)\left(z_{1}, z_{2}\right)= & \frac{b_{n} \psi_{n, q_{1}}^{1}\left(z_{1}\right)}{2\left(1+a_{n} b_{n}\right)} \frac{\partial f}{\partial z_{1}}\left(z_{1}, z_{2}\right)+\frac{b_{n} \psi_{n, q_{2}}^{1}\left(z_{2}\right)}{2\left(1+a_{n} b_{n}\right)} \frac{\partial f}{\partial z_{2}}\left(z_{1}, z_{2}\right) \\
& -\frac{b_{n} \psi_{n, q_{2}}^{1}\left(z_{2}\right) \psi_{n, q_{1}}^{1}\left(z_{1}\right)}{2\left(1+a_{n} b_{n}\right)} \frac{\partial^{2} f}{\partial z_{2} \partial z_{1}}\left(z_{1}, z_{2}\right) \\
& -\frac{b_{n} \psi_{n, q_{2}}^{1}\left(z_{2}\right) \psi_{n, q_{1}}^{2}\left(z_{1}\right)}{4\left(1+a_{n} b_{n}\right)} \frac{\partial^{3} f}{\partial z_{2} \partial z_{1}^{2}}\left(z_{1}, z_{2}\right) \\
& -\frac{b_{n} \psi_{n, q_{2}}^{2}\left(z_{2}\right) \psi_{n, q_{1}}^{1}\left(z_{1}\right)}{4\left(1+a_{n} b_{n}\right)} \frac{\partial^{3} f}{\partial z_{2}^{2} \partial z_{1}}\left(z_{1}, z_{2}\right) \\
& -\frac{b_{n} \psi_{n, q_{2}}^{2}\left(z_{2}\right) \psi_{n, q_{1}}^{2}\left(z_{1}\right)}{8\left(1+a_{n} b_{n}\right)} \frac{\partial^{4} f}{\partial z_{2}^{2} \partial z_{1}^{2}}\left(z_{1}, z_{2}\right),
\end{aligned}
$$

and

$$
K_{n}(f)\left(z_{1}, z_{2}\right)=\frac{b_{n}}{4\left(1+a_{n} b_{n}\right)}\left\{\psi_{n, q_{1}}^{2}\left(z_{1}\right) \frac{\partial^{2} f}{\partial z_{1}^{2}}\left(z_{1}, z_{2}\right)+\psi_{n, q_{2}}^{2}\left(z_{2}\right) \frac{\partial^{2} f}{\partial z_{2}^{2}}\left(z_{1}, z_{2}\right)\right\} .
$$

Considering Theorems 2 and 3 in [19], we get

$$
\lim _{n \rightarrow \infty} E_{n}(f)\left(z_{1}, z_{2}\right)=0 \quad \text { and } \quad \lim _{n \rightarrow \infty} F_{n}(f)\left(z_{1}, z_{2}\right)=0
$$

Under the conditions of the theorem, since $\lim _{n \rightarrow \infty} a_{n}=0, \lim _{n \rightarrow \infty} \frac{1}{b_{n}}=0, \lim _{n \rightarrow \infty} a_{n} \times$ $b_{n}=0$ for $0<\beta<\frac{1}{2}$, it is also clear that

$$
\lim _{n \rightarrow \infty} G_{n}(f)\left(z_{1}, z_{2}\right)=0 .
$$

From Theorem 2, we obtain

$$
\lim _{n \rightarrow \infty}\left\|2\left(a_{n}+\frac{1}{b_{n}}\right)\left[\frac{D_{n}(f)}{4\left(a_{n}+\frac{1}{b_{n}}\right)^{2}}\right]+E_{n}(f)+F_{n}(f)+G_{n}(f)\right\|_{r_{1}, r_{2}}=0 .
$$

Using $\lim _{n \rightarrow \infty} a_{n} b_{n}=0$ for $0<\beta<\frac{1}{2}$ and $\frac{1}{1+a_{n}\left|z_{1}\right|} \geq \frac{2}{3}$, we get

$$
\left\|K_{n}(f)\right\|_{r_{1}, r_{2}} \geq \frac{1}{18\left(1+a_{n} b_{n}\right)}\left|z_{1}\right|\left|\frac{\partial^{2} f}{\partial z_{1}^{2}}\left(z_{1}, z_{2}\right)\right| .
$$


Similarly, it follows that

$$
\left\|K_{n}(f)\right\|_{r_{1}, r_{2}} \geq \frac{1}{18\left(1+a_{n} b_{n}\right)}\left|z_{2}\right|\left|\frac{\partial^{2} f}{\partial z_{2}^{2}}\left(z_{1}, z_{2}\right)\right| .
$$

From equations (14) and (15), we can write

$$
\left\|K_{n}(f)\right\|_{r_{1}, r_{2}} \geq \frac{1}{36\left(1+a_{n} b_{n}\right)}\|K(f)\|_{r_{1}, r_{2}} .
$$

In equation (13), taking into account the inequalities

$$
\|H+T\|_{r_{1}, r_{2}} \geq\left|\|H\|_{r_{1}, r_{2}}-\|T\|_{r_{1}, r_{2}}\right| \geq\|H\|_{r_{1}, r_{2}}-\|T\|_{r_{1}, r_{2}},
$$

and equation (16), it follows that

$$
\begin{aligned}
\left\|R_{n, n}^{q_{1}, q_{2}}(f)-f\right\|_{r_{1}, r_{2}} \geq & 2\left(a_{n}+\frac{1}{b_{n}}\right)\left\{\left\|K_{n}(f)\right\|_{r_{1}, r_{2}}\right. \\
& \left.-\left\|2\left(a_{n}+\frac{1}{b_{n}}\right)\left[\frac{D_{n}(f)}{4\left(a_{n}+\frac{1}{b_{n}}\right)^{2}}\right]+E_{n}(f)+F_{n}(f)+G_{n}(f)\right\|_{r_{1}, r_{2}}\right\} \\
\geq & \left(a_{n}+\frac{1}{b_{n}}\right)\left\|K_{n}(f)\right\|_{r_{1}, r_{2}} \\
\geq & \left(a_{n}+\frac{1}{b_{n}}\right) \frac{1}{36\left(1+a_{n} b_{n}\right)}\|K(f)\|_{r_{1}, r_{2}}
\end{aligned}
$$

for all $n \geq n_{0}$ with $n_{0}$ depending only $f, r_{1}$ and $r_{2}$. We used that by hypothesis we have $\|K(f)\|_{r_{1}, r_{2}}>0$.

Combining Theorem 2 with Theorem 3, we immediately obtain the following result giving the exact degree of the operators (1).

Corollary 1 Suppose that the hypothesis in the statement of Theorem 3 holds. If the Taylor series off contains at least one term of the form $c_{k, 0} z_{1}^{k}$ with $c_{k, 0} \neq 0$ and $k \geq 2$ or of the form $c_{0, j} z_{2}^{j}$ with $c_{0, j} \neq 0$ and $j \geq 2$, then for all $n \geq n_{0}$ we have

$$
\left\|R_{n, n}^{q_{1}, q_{2}}(f)-f\right\|_{r_{1}, r_{2}} \sim\left(a_{n}+\frac{1}{b_{n}}\right) .
$$

Proof It suffices to prove that, under the hypothesis on $f$, it cannot be a solution of the complex partial differential equation

$$
z_{1} \frac{\partial^{2} f}{\partial z_{1}^{2}}\left(z_{1}, z_{2}\right)+z_{2} \frac{\partial^{2} f}{\partial z_{2}^{2}}\left(z_{1}, z_{2}\right)=0, \quad\left|z_{1}\right|<R_{1},\left|z_{2}\right|<R_{2} .
$$

Indeed, suppose the contrary. Since a simple calculation gives

$$
\begin{aligned}
z_{1} \frac{\partial^{2} f}{\partial z_{1}^{2}}\left(z_{1}, z_{2}\right)+z_{2} \frac{\partial^{2} f}{\partial z_{2}^{2}}\left(z_{1}, z_{2}\right)= & \sum_{k=1}^{\infty} c_{k+1,0} k(k+1) z_{1}^{k}+\sum_{k=1}^{\infty} c_{k+1,1} k(k+1) z_{1}^{k} z_{2} \\
& +2 \sum_{j=2}^{\infty} c_{2, j} z_{1} z_{2}^{j}+\sum_{k=2}^{\infty} \sum_{j=2}^{\infty} c_{k+1, j} k(k+1) z_{1}^{k} z_{2},
\end{aligned}
$$




$$
\begin{aligned}
& +\sum_{j=1}^{\infty} c_{0, j+1} j(j+1) z_{2}^{j}+\sum_{j=1}^{\infty} c_{1, j+1} j(j+1) z_{1} z_{2}^{j} \\
& +2 \sum_{k=2}^{\infty} c_{k, 2} z_{1}^{k} z_{2}+\sum_{k=2}^{\infty} \sum_{j=2}^{\infty} c_{k, j+1} j(j+1) z_{1}^{k} z_{2}^{j}
\end{aligned}
$$

by setting equal to zero and by the identification of the coefficients, from the terms under the first and fifth sign $\sum$, we immediately get $c_{k+1,0}=c_{0, j+1}=0$, for all $k=1,2, \ldots$ and $j=$ $1,2, \ldots$, which contradicts the hypothesis on $f$. Therefore the hypothesis and the lower estimate in Theorem 3 are satisfied, which completes the proof.

\section{Competing interests}

I declare that I have no competing interests.

\section{Acknowledgements}

The author is grateful to the editor and the reviewers for making valuable suggestions, leading to a better presentation of the work.

\section{Received: 21 September 2013 Accepted: 12 December 2013 Published: 14 Jan 2014}

\section{References}

1. Ostrovska, S: On the q-Bernstein polynomials and theirs iterates. Adv. Stud. Contemp. Math. 11, $193-204$ (2005)

2. Gal, SG, Gupta, V, Mahmudov, NI: Approximation by a complex q-Durmeyer type operator. Ann. Univ. Ferrara 58, 65-87 (2012)

3. Agarwal, RP, Gupta, V: On q-analogue of a complex summation-integral type operators in compact disks. J. Inequal. Appl. 2012, 111 (2012)

4. Mahmudov, NI: Convergence properties and iterations for $q$-Stancu polynomials in compact disks. Comput. Math. Appl. 59(12), 3763-3769 (2010)

5. Gal, SG: Approximation in compact sets by $q$-Stancu-Faber polynomials, $q>1$. Comput. Math. Appl. 61(10), 3003-3009 (2011)

6. Gal, SG: Voronovskaja's theorem, shape preserving properties and iterations for complex $q$-Bernstein polynomials. Studia Sci. Math. Hung. 48(1), 23-43 (2011)

7. Gal, SG: Approximation by $q$-Lorentz polynomials, $q>1$. Mathematica 54(77), 53-63 (2012)

8. Gal, SG: Overconvergence in Complex Approximation. Springer, New York (2013)

9. Andrews, GE, Askey, R, Roy, R: Special Functions. Cambridge University Press, Cambridge (1999)

10. Kac, V, Cheung, P: Quantum Calculus. Springer, New York (2002)

11. Aral, A, Gupta, V, Agarwal, RP: Applications of q Calculus in Operator Theory. Springer, New York (2013). ISBN:978-1-4614-6945-2

12. Balázs, K: Approximation by Bernstein type rational function. Acta Math. Acad. Sci. Hung. 26, 123-134 (1975)

13. Balázs, K, Szabados, J: Approximation by Bernstein type rational function Il. Acta Math. Acad. Sci. Hung. 40(3-4), 331-337 (1982)

14. Dogru, O: On statistical approximation properties of Stancu type bivariate generalization of q-Balazs-Szabados operators. In: Proceedings. Int. Conf on Numerical Analysis and Approximation Theory, Cluj-Napoca, Romania, pp. 179-194 (2006)

15. Atakut, Ç, İspir, N: On Bernstein type rational functions of two variables. Math. Slovaca 54, 291-301 (2004)

16. Gupta, V, Ispir, N: On the Bézier variant of generalized Kantrovich type Balazs operators. Appl. Math. Lett. 18(9), 1053-1061 (2005)

17. Gal, SG: Approximation by Complex Bernstein and Convolution Type Operators. World Scientific, New Jersey (2009)

18. Yıldız Özkan, E: Approximation by complex bivariate Balázs-Szabados operators. Bull. Malays. Math. Soc. (accepted)

19. İspir, N, Yıldız Özkan, E: Approximation properties of complex q-Balazs-Szabados operators in compact disks. J. Inequal. Appl. 2013, 361 (2013)

10.1186/1029-242X-2014-20

Cite this article as: Yıldız Özkan: Approximation properties of bivariate complex $q$-Balàzs-Szabados operators of tensor product kind. Journal of Inequalities and Applications 2014, 2014:20 